Meta

Journal des tradlucteurs

Translators' Journal

\title{
Quelques remarques sur les adverbes français en -ment et leur rapport sur les adverbes anglais en -ly
}

\section{Chantal Bertrand}

Volume 31, numéro 2, juin 1986

URI : https://id.erudit.org/iderudit/002066ar

DOI : https://doi.org/10.7202/002066ar

Aller au sommaire du numéro

Éditeur(s)

Les Presses de l'Université de Montréal

ISSN

0026-0452 (imprimé)

1492-1421 (numérique)

Découvrir la revue

Citer cet article

Bertrand, C. (1986). Quelques remarques sur les adverbes français en -ment et leur rapport sur les adverbes anglais en -ly. Meta, 31(2), 179-203.

https://doi.org/10.7202/002066ar d'utilisation que vous pouvez consulter en ligne.

https://apropos.erudit.org/fr/usagers/politique-dutilisation/ 


\section{QUELQUES REMARQUES SUR LES ADVERBES FRANCAIS EN -MENT ET LEUR RAPPORT AVEC LES ADVERBES ANGLAIS EN -LY*}

\section{INTTRODUCTION}

Dans un article, K. Connors (1973) tente, dans le cadre d'un projet de traduction automatique, de désambiguïser les adverbes en $-l y$ les plus fréquents ${ }^{1}$ en établissant pour chaque fonction adverbiale une position syntaxique préférentielle. Ce procédé permet une traduction "probabiliste" des adverbes en -ly. Les adverbes sont classés par fonction; aucune traduction n'en est donnée. Pour la traduction des adverbes, nous avons dès lors eu recours au dictionnaire Robert-Collins (1981).

L'article de $\mathbf{K}$. Connors soulève plusieurs problèmes importants, notamment : - le fait qu'il n'existe pas d'adverbe en -ment pour chaque adverbe en -ly examiné. Il s'agit donc d'essayer de déterminer si l'absence d'adverbes en -ment est morphologique, lexicale ou les deux à la fois ;

- même lorsque l'adverbe en -ment existe, il n'est pas toujours possible de l'utiliser. Ainsi, des informateurs préferent $c$ ) à $b$ )

a) He acted naturally

b) Il a joué naturellement

c) Il a joué de façon naturelle

Nous nous proposons d'examiner brièvement chacun des deux points ci-dessus, de façon à mettre en évidence certaines différences entre le français et l'anglais. Même si les résultats sont incomplets et discutables, nous espérons cependant montrer l'intérêt de ce type de recherche pour les traducteurs.

\section{LA RÈGLE DE FORMATION DE L'ADVERBE EN -MENT}

Tardif (1982:149) propose la règle suivante pour la formation des adverbes en -ment :

$[\mathrm{X}]_{\mathrm{adj} .} \longrightarrow[\mathrm{X}]+$ ment $]_{\text {adv }}^{2}$

Le même auteur propose une règle équivalente pour la formation des adverbes en ly :

$\left.[\mathrm{X}]_{\mathrm{adj} .} \longrightarrow[\mathrm{X}]+1 \mathrm{y}\right]_{\mathrm{adv}}$.

Sabourin (1977 : 17) fait remarquer que le corpus anglais de l'Université Brown comporte plus de 12000 adverbes en -ly. Or, seulement 1257 adverbes en -ment figurent dans le Dictionnaire inverse de la langue française (dorénavant DIF) de Juilland $(1965)^{3}$. Puisque la règle de formation des deux types d'adverbes est la même, on peut se demander pourquoi les adverbes en -ly sont tellement plus nombreux. La réponse à cette question implique une recherche morphologique approfondie, ce qui sort des limites du présent article. Une étude préliminaire nous a toutefois permis de constater que l'anglais, contrairement au français, semble avoir des règles productives de formation d'adjectif (et donc la possibilité de former plus d'adverbes). À ce problème du "nombre d'adjectifs ", s'ajoute le fait qu'en français, la base des adverbes en -ment semble soumise à une contrainte morphologique, ainsi qu'à des contraintes sémantiques. De plus, certaines restrictions limitent l'emploi des adverbes en -ment : la non-lexicalisation de certains adverbes et l'utilisation de l'adjectif adverbialisé (c'est-à-dire invariable et intransportable), voire de noms adverbialisés, au lieu de l'adverbe dérivé.

Lorsqu'on examine ce problème sous l'angle de la traduction, une contrainte supplémentaire apparaît : l'adverbe en -ly ne peut être traduit par l'adverbe en -ment que si les deux adverbes ont la même " fonction ", c'est-à-dire appartiennent à la même classe sémantique. Dans le cas inverse, le français doit recourir à un syntagme prépositionnel 
(S.Prép.), à un syntagme nominal (SN) ou à une proposition. Cette contrainte "fonctionnelle " sera discutée plus loin.

\subsection{Contrainte morphologique}

L'adjectif qui sert de base à l'adverbe en -ment ne peut pas être un déverbal ${ }^{4}$ se terminant par [mã] : *charmamment : de façon charmante ; *alarmamment : de façon alarmante; *calmamment : de façon calmante.

Il ne semble pas non plus y avoir d'adverbe en -ment formé sur une base se terminant en [nã] ${ }^{5}$ ou en [jã] : *ricanamment : de façon ricanante, ${ }^{*}$ gênamment : de façon gênante, *surprenamment : de façon surprenante, *stagnamment : de façon stagnante. Les adverbes en -ment ne semblent donc pas admettre une base déverbale se terminant par une nasale

D'autre part, la règle de formation fait appel, dans les deux langues, à une base adjectivale. Le français ne semble pas avoir de règle productive de formation d'adjectif. L'anglais, par contre, semble avoir une règle productive de formation d'adjectifs à partir de la forme en ing (cf. surprisingly) et à partir de noms suffixés en -ful ou -ness : helpful : helpfully, helpness : helplessly, harmful : harmfully, harmless : harmlessly.

Le seul processus productif permettant, en français, d'obtenir un adverbe à partir d'un nom n'existe en principe qu'en français populaire ${ }^{7}$. Ceci a été mis en évidence par L. Drapeau (1982 : 29-30) :

a) Il marche tapette (*tapettement)

b) Oui, oui, puis ils parlaient habitant... (Sankoff et Cedergren $1967: 663$ )

c) Il mange cochon (*cochonnement)

d) Il chauffe colon (* colonnement) comme un sunday-driver

f). Dans les grandes villes, le monde vit robot (*robotement) pas mal.

\subsection{Contraintes sémantiques}

Certains adjectifs ne peuvent pas servir de base aux adverbes en -ment. Ce sont des adjectifs désignant des qualités perceptibles par les sens ${ }^{8}$, des adjectifs désignant des types ou des espèces.

\subsubsection{Adjectifs désignant une qualité perceptible par les sens}

Au sens propre (par opposition à sens figuré), le français n'a pas les adverbes suivants : *rougement (aucun adjectif de couleur n'a d'adverbe en -ment correspondant sauf vertement employé au figuré $e^{8}$; ${ }^{*}$ jeunement ; *prochement ; *vieillement ; *lointainement ; grossement ; *mincement.

Les exceptions sont rares et à part joliment (qui peut être utilisé au sens propre ou comme intensificateur), il s'agit chaque fois d'adverbes utilisés dans un sens métaphorique.

a) Pierre a été maigrement payé.

b) Pierre a été grassement payé.

L'affaire a été rondement menée.

C'est carrément faux.

Comment vas-tu? Petitement.

Certains adverbes de ce type ont existé. Ils ont toutefois disparu ou sont en voie de disparition ${ }^{10}$.

\subsubsection{Adjectifs désignant des types ou des espèces}

Les adjectifs désignant des types ou des espèces ne peuvent pas servir de base aux adverbes en -ment. Ceci est noté par F. Labelle (1974: 34) qui, sans prétendre établir 
une liste exhaustive, cite les adjectifs suivants : animal, glaciaire, canin, protéique, chevalin, vestimentaire.

À première vue, les deux contraintes sémantiques ci-dessus sont très normales si l'on accepte l'hypothèse que l'adverbe en -ment est d'abord un adverbe de manière. L'adjectif qui lui sert de base doit donc être un "mot descriptif » désignant une façon d'agir ou de penser, mais pas un état.

Il y aurait lieu de vérifier dans quelle mesure les adverbes en -ly sont soumis à des contraintes sémantiques.

\subsection{Irrégularités paradigmatiques du français}

Nous examinons ici deux types d'irrégularités paradigmatiques des adverbes en -ment :

- le fait qu'un grand nombre d'adverbes négatifs préfixés en in-n'aient pas de correspondant positif ou l'inverse ;

- le fait qu'un grand nombre d'adverbes morphologiquement (et sémantiquement) possibles n'aient jamais été formés. C'est ce que l'on appelle communément des lacunes dérivationnelles.

\subsubsection{Le problème posé par le préfixe négatif in-}

Certains adverbes en -ment existent à la fois sous la forme positive et sous la forme négative. La forme négative est généralement formée au moyen du préfixe in 11 $^{11}$ À quelques exceptions près ${ }^{12}$, adverbe positif et adverbe négatif sont alors de sens contraire : consciemment : inconsciemment, décemment : indécemment, pudiquement : impudiquement, modérément : immodérément.

Toutefois, un grand nombre d'adverbes négatifs n'ont pas de correspondant positif (64 adverbes dans le DIF). Ex. : infatigablement : * fatigablement ${ }^{13}$, incroyablement : *croyablement, impudemment : *pudemment.

Inversement, certains adverbes positifs n'ont pas de correspondant négatif. Ex. : sciemment : *insciemment, glorieusement : *inglorieusement, commodément : *incommodément.

Nous ne discuterons pas ici les implications morphologiques de cette situation. Nous voulons simplement souligner le fait que l'irrégularité du paradigme limite le nombre d'adverbes en -ment.

D'après les données de M. Picard (1982 : 191), la situation est la même en anglais, à une grande différence près, c'est que le préfixe in-ne serait pas le préfixe négatif par ex.cellence. Logiquement, l'irrégularité paradigmatique des adverbes en -ly devrait donc être beaucoup moins forte.

\subsubsection{Lacunes morphologiques}

Beaucoup d'adverbes en -ment morphologiquement et sémantiquement possibles n'ont jamais été formés. En parcourant le DIF, on constate particulièrement les lacunes suivantes :

- Ainsi que cela a été signalé, beaucoup de participes présents n'ont pas d'adverbes en -ment correspondant, même lorsque la contrainte morphologique n'est pas en cause. Ex. : *fracassamment : de façon fracassante, *écrasamment : de façon écrasante, *accablamment : de façon accablante.

Le DIF comporte plusieurs pages de ces adjectifs déverbaux. Les seules exceptions que nous ayons relevées sont : courant : couramment (courir), incessant : incessamment (cesser), précipitant : précipitamment (précipiter). 
Beaucoup de participes passés n'ont pas non plus d'adverbe correspondant. Ex. : *favorisément : avec favoritisme, *détaillément : de façon détaillée, *excitément : de façon excitée, *têtutement : avec entêtement.

L'anglais, par contre, semble pouvoir former très librement des adverbes en -ly à partir de participes présents ou passés. Ex. : seemingly, allegedly, sparingly, supposedly, increasingly, markedly.

Le nombre d'adverbes en -ment est donc d'autant plus limité que beaucoup d'adverbes en -ment n'ont jamais été lexicalisés (ce qui est particulièrement le cas des adverbes déverbaux) et que le français n'a pas de règle productive de formation d'adjectif.

On peut alors se demander ce qui remplace l'adverbe en -ment non lexicalisé. La réponse est double : le syntagme prépositionnel et l'adjectif adverbialisé (français écrit et oral), voire le nom adverbialisé (oral).

Nous allons successivement examiner le rôle du syntagme prépositionnel et celui de l'adjectif adverbialisé.

\subsection{Rapports entre le syntagme prépositionnel et l'adverbe en -ment}

Le syntagme prépositionnel est toujours disponible. Autrement dit, le syntagme prépositionnel est, en français, en concurrence constante avec l'adverbe en -ment.

Dans certains cas, le syntagme prépositionnel est préféré ; c'est le cas quand l'adverbe en -ment est ambigu. Ex. :

a) He acted naturally

b) Il a joué naturellement

c) Il a joué de façon naturelle

d) Il a joué très naturellement

Ainsi que nous le verrons, l'exemple (b) est ambigu ; il est en effet paraphrasable par:

Il a joué de façon naturelle (manière)

Naturellement, (bien sûr), il a joué (adverbe de phrase).

Par contre les exemples $c$ ) et $d$ ) ne sont pas ambigus : le syntagme prépositionnel ne permet que l'interprétation "manière ". Or, l'adverbe occupe dans $d$ ) la même position syntaxique que dans $b$ ). La seule différence est que dans $d$ ) l'adverbe est intensifié. Tout semble donc se passer comme si le sens "manière " s'affaiblissait lorsque l'adverbe a plus d'une fonction et qu'il occupe une position ambiguë. La façon la plus simple (et la plus courante) de désambiguïser la phrase consiste à utiliser le syntagme prépositionnel au lieu de l'adverbe : on peut également intensifier l'adverbe ambigu. On peut vérifier ce fait avec les exemples suivants :

a) Pierre l'a expliqué précisément

b) Pierre l'a expliqué de façon précise

c) Pierre l'a expliqué très précisément

d) Pierre l'a dit franchement

e) Pierre l'a dit de façon franche

f) Pierre l'a dit bien (très) franchement

Le syntagme prépositionnel est également obligatoire lorsqu'il s'agit de traduire un adverbe en -ly ayant une fonction que son "sosie" en -ment n'a pas.

\subsection{Rapports entre l'adjectif adverbialisé et l'adverbe en -ment}

L'adjectif adverbialisé est un adjectif employé comme adverbe. Cet " adjectif » devient alors invariable et intransportable (c'est-à-dire qu'il ne peut occuper qu'une seule position dans la phrase, contrairement à la plupart des adverbes en -ment). Le français standard ne comprend qu'un petit nombre de ces adjectifs. Ex. : crier fort; parler bas ; 
chanter faux; marcher droit ; pousser dru; boire sec, boire ferme; couper ras; voir clair ; filer doux.

L. Drapeau (1982 : 17-21) fait remarquer que ce processus est beaucoup plus productif en français populaire qu'en français standard. L'auteur montre qu'il s'agit d'un moyen de combler les lacunes de la suffixation en -ment et d'utiliser des emprunts non intégrés. Voici quelques-uns des exemples de cet auteur : Il agit rusé ; il danse " pogné "; ils jouent groupé ; il écrit tourmenté ; il marche tendu ; elle chante straight (*straightement); les Noirs dansent cool (*coolement); il marche flyé (*flyément).

L'utilisation du syntagme prépositionnel ou de l'adjectif adverbialisé limite également le nombre d'adverbes en -ment.

En résumé, nous avons constaté que le nombre d'adverbes en -ment est limité par :

- une contrainte morphologique,

- l'absence d'une règle productive de formation d'adjectifs,

des contraintes sémantiques.

- des irrégularités paradigmatiques,

- la concurrence du syntagme prépositionnel et de l'adjectif adverbialisé.

Contrairement au français, l'anglais ne semble pas présenter de restrictions quant à la formation d'adverbes déverbaux. En outre, l'anglais semble pouvoir former des adverbes en -ly à partir d'adjectifs dénominaux. Ces deux facteurs expliquent partiellement pourquoi il y a beaucoup plus d'adverbes en -ly que d'adverbes en -ment. À ces deux facteurs il convient d'ajouter les restrictions relatives à la formation, la lexicalisation et l'emploi des adverbes en -ment.

Sur les 322 adverbes en -ly répertoriés par K. Connors, 63 adverbes n'ont pas de correspondant en -ment soit $19,56 \%$, huit autres correspondent plus ou moins à des adverbes en -ment en ce sens qu'une traduction au moins est un adverbe en -ment (voir annexe III).

\section{COMPARAISON DES ADVERBES EN -MENT ET DES ADVERBES EN -LY DU POINT DE VUE FONCTIONNEL}

Dans cette partie, nous examinons les adverbes du point de vue de leur fonction, c'est-à-dire du point de vue de la catégorie sémantique à laquelle ils appartiennent.

\subsection{Fonctions et positions syntaxiques des adverbes en -ment}

D'après l'analyse de F. Jakendoff (1972) : $47 \mathrm{sq}$.) et sur la base du critère d'antéposition possible à une phrase niée, F. Labelle (1974 : 14 sq.) distingue deux grandes catégories d'adverbes : les adverbes de phrase et les adverbes de syntagme verbal (SV).

Les adverbes de phrase, contrairement aux adverbes de SV, peuvent être antéposés à une phrase niée. Ex. : Évidemment, Jacques n'est pas venu (adv. de phrase).

Ces adverbes sont introduits sous le noud $\mathbf{P}$ et son [+ mobiles], c'est-à-dire qu'ils peuvent occuper toutes les positions où ils sont directement dominés par le nœud $\mathrm{P}$. Ils peuvent donc occuper les positions 1-2A-4 et 5 du schéma de la page suivante. Ils ne peuvent pas occuper la position 3 .

Ex. : (Évidemment) Cornélie (évidemment) a (évidemment) vendu *(évidemment) son vélocipède (évidemment) $)^{14}$

L'adverbe évidemment pourrait également figurer en position 5, c'est-à-dire après en S.Prép. : Cornélie a vendu son vélocipède à Pierre(,) évidemment.

Les adverbes de SV ne peuvent pas être antéposés à une phrase niée. Ex. :

a) manière : *Efficacement, il n'a pas résolu le problème.

b) intensificateur : *Énormément, il n'a pas résolu le problème.

Ces adverbes sont introduits sous le ncud SV et ont une mobilité limitée (ils ne peuvent pas être déplacés à gauche du morphème porteur de la marque du temps et ne 


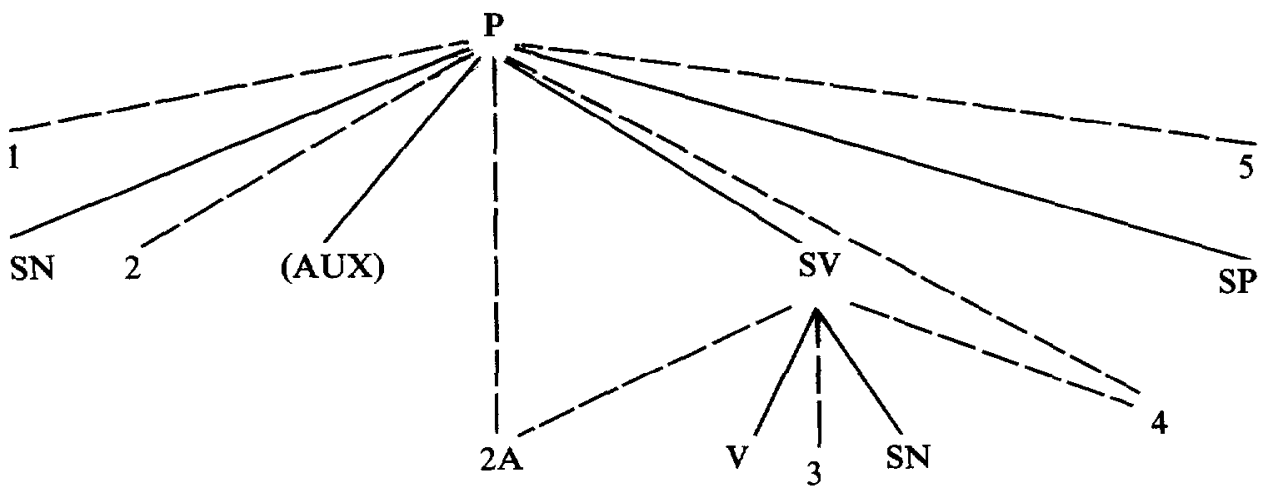

peuvent pas non plus être déplacés à gauche de l'auxiliaire). Les adverbes de SV peuvent occuper les positions $2 \mathrm{~A}, 3,4$ du schéma ci-dessus. Ils ne peuvent pas occuper les positions 1,2 et 5 . Ex. :

a) *(Harmonieusement) Bosch *(harmonieusement) a (harmonieusement) agencé (harmonieusement) ses couleurs (harmonieusement).

b) *Cornélie a vendu son vélocipède à Pierre harmonieusement.

c) *Cornélie a vendu son vélocipède à Pierre énormément:

Certains adverbes peuvent être introduits soit sous $S V$, soit sous $P$, d'où leur ambiguité ou leur changement de sens. Les positions $2 \mathrm{~A}$ (entre l'auxiliaire et le verbe) et 4 (après l'objet direct SN) seront ambiguës pour tout adverbe de ce type. Nous reviendrons ultérieurement à ces problèmes d'ambiguïté.

\subsubsection{Les adverbes de phrase}

Labelle (1974) utilise le terme « adverbe de phrase » dans un sens très large. En effet, ces adverbes comprennent quatre sous-groupes (la terminologie, les exemples et les paraphrases sont de Labelle).

a) Les adverbes à valeur modale

Étant donné l'exemple proposé, le terme modal semble référer ici à modalité, au sens où l'on entend modalité affirmative, négative et interrogative. Ce type d'adverbe donne en fait l'opinion du locteur. Ex. :

Évidemment, Adolphe n'a pas fait son devoir.

b) Les adverbes orientés vers le sujet

Stupidement, Adolphe n'a pas parlé au ministre. (Paraphrase : il est stupide de la part d'Adolphe de ne pas avoir parlé au ministre.)

c) Les adverbes de point de vue

Médicalement, Adolphe n'est pas mort. (Paraphrase : au point de vue médical, Adolphe n'est pas mort.)

d) Les adverbes de temps

Habituellement, Albert ne fait pas son devoir.

\subsubsection{Les adverbes de $S V$}

Les adverbes de SV comprennent deux groupes : les adverbes de manière (dont la paraphrase est "d'une manière Adj") et les adverbes d'intensité. 
Selon Labelle (1974 : 27), le critère d'acceptabilité dans la chaîne : il est ADV ADJ permet de séparer les deux sous-catégories ; l'adverbe de manière ne peut pas être inséré dans cette séquence, contrairement à l'adverbe d'intensité. Ex. :

$a d v$. de manière : ${ }^{*}$ Ce technicien est aisément (efficacement) qualifié.

$a d v$. d'intensité : Ce technicien est hautement qualifié.

En pratique, il est souvent difficile de déterminer si un adverbe donné a le sens manière ou celui d'intensité. Ex. : Être douloureusement blessé.

\subsubsection{Les problèmes d'ambigüté}

Ainsi que nous l'avons signalé, les positions 2A (entre l'auxiliaire et le verbe) et 4 (après l'objet SN) seront ambiguës pour tout adverbe possédant à la fois l'interprétation adverbe de phrase et adverbe de SV. (Cf. Labelle (1974 : 30) : point de vue / manière, manière / adverbe à valeur modale, manière / temps, manière / adv. orienté vers le sujet.)

L'exemple "Il a joué naturellement montre l'ambiguité manière / adverbe à valeur modale.

\subsubsection{Les adverbes de phrase sous $\bar{P}$}

Outre les adverbes de phrase mentionnés en 2.1.1, le français possède un type d'adverbe de phrase que C. Dubuisson (1975:32) représente comme dominé par le nœud $\overline{\mathbf{P}}$. Les adverbes ci-dessous sont des adverbes de ce type :

$\left.\begin{array}{l}\text { Naturellement } \\ \text { Évidemment } \\ \text { Certainement } \\ \text { Assurément } \\ \text { Peut-être } \\ \text { Heureusement } \\ \text { Apparemment } \\ \text { Probablement } \\ \text { Vraisemblablement } \\ \text { ? Possiblement (Québec) }\end{array}\right)$ que Marie viendra

C. Dubuisson (1975 : 32) propose, pour ces adverbes, la représentation suivante :



Il semble s'agir d'un nombre restreint d'adverbes indiquant la certitude ou la probabilité. En tout cas, l'adverbe est en quelques sorte "positif». Ex. : *Malheureusement que Pierre viendra. Ce type d'adverbe n'existe pas en anglais. 


\subsection{Fonctions des adverbes en -ly}

K. Connors (1973:29 sq.) postule quatre types d'adverbes : les adverbes de manière, les adverbes de temps, les adverbes de phrase, les adverbes "d'extension " $(E X$ TENT), ce qui comprend les adverbes de domaine et les adverbes d'intensité.

\subsubsection{L'adverbe de manière}

L'adverbe de manière a la même paraphrase qu'en français : $d^{\prime}{ }^{\prime}$ ne manière $A d j \cdot{ }_{x}$. Cet adverbe cumule souvent deux fonctions : la manière et l'« extension ».

Les adverbes n'ayant que la fonction manière sont les moins fréquents.

\subsubsection{L'adverbe de temps}

L'adverbe de temps n'est pas défini. Il est toutefois précisé par des traits de fréquence ('frequency'), orientation dans le temps ('forward-/backward-looking'), de durée ('durative').

Ces traits s'avèrent précieux pour comparer les adverbes en - $l y$ et les adverbes en -ment. Dans certains cas, la fonction est la même, mais le trait "secondaire » diffère. L'organisation des traits est la suivante :

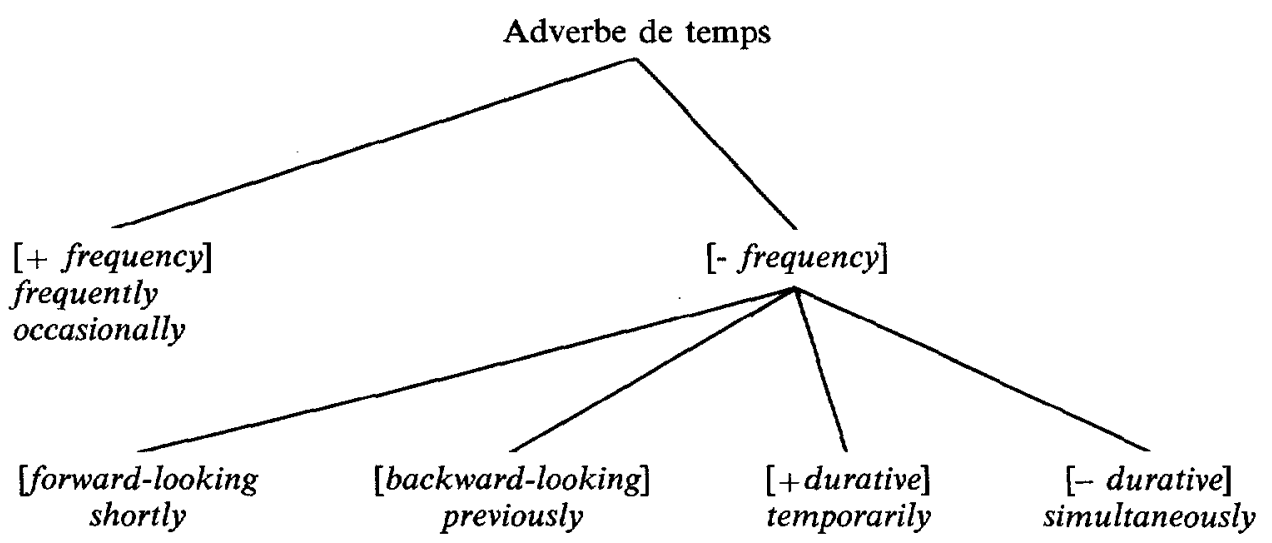

L'adverbe de temps n'a que la manière comme autre fonction possible.

\subsubsection{L'adverbe de phrase}

Est adverbe de phrase tout Adverbe ${ }_{\mathrm{x}}$ qui correspond à un adjectif et dont la paraphrase est : "Il est Adj. que... " (K. Connors 1973 : 32) : "Naturally, he was nervous."

L'adverbe de phrase peut avoir comme fonctions la manière et l'extension.

\subsubsection{Les adverbes d'extension}

Les adverbes d'extension se subdivisent en adverbes de degré, dont la paraphrase est : "A un Adj.x degré ", et adverbes de domaine, dont la paraphrase est : " Dans le domaine de l'Adj. . "

Les adverbes de degré correspondent à ce que Labelle appelle adverbes d'intensité, adverbes plus couramment connus en linguistique sous le nom d'intensificateurs, appellation que nous adoptons.

Les adverbes de domaine correspondent en gros à ce que Labelle appelle adverbes de point de vue, appellation que nous adoptons. Les catégories domaine et point de vue ne se recoupent cependant pas tout à fait. 
En anglais, les adverbes de domaine sont en distribution quasi complémentaires avec les adverbes de phrase. Un adverbe de point de vue peut avoir deux autres fonctions : il peut être adverbe de manière ou adverbe de phrase.

\subsection{Quelques problèmes de terminologie}

Toute tentative de mise en parallèle de travaux de chercheurs différents se heurte invariablement à des problèmes terminologiques. Or, toute comparaison suppose que l'on compare bien les mêmes éléments. Deux catégories adverbiales diffèrent d'une analyse à l'autre : l'adverbe de phrase et l'adverbe de domaine.

\subsubsection{L'adverbe de phrase}

En français, l'adverbe de phrase accepte généralement la paraphrase : "Il est Adj.x que... ». Ex. :

a) Évidemment, Jean est (n'est pas) venu.

b) Il est évident que Jean est (n'est pas venu. "

Toutefois, ainsi que le signale C. Sabourin $(1975: 71)$, cette paraphrase a une double limite :

d'une part, certains adverbes qui ne sont pas des adverbes de phrase acceptement la paraphrase en question. Ex. : *Abominablement, Jean est (n'est pas) venu.

a) Jean a agi abominablement

b) Il est abominable que Jean ait agi de pareille façon.

- d'autre part, certains adverbes de phrase n'acceptent pas d'être paraphrasés ainsi. Ex. : Effectivement, Jean est (n'est pas) venu.

*Il est effectif que Jean est venu.

En fait, cette deuxième limite est signalée pour l'anglais par R. Jackendoff (1972: 51) pour des adverbes qui, dans la terminologie de Labelle (1974), seraient des adverbes de phrase de type modal.

Nous considérons donc la paraphrase comme peu sûre. D'autre part, la définition de l'adverbe de phrase selon K. Connors (1973) est trop stricte puisqu'il y a des lacunes dérivationnelles aussi bien en français qu'en anglais : aucune des deux langues n'a systématiquement un adjectif pouvant servir de «source » à l'adverbe de phrase correspondant. Pour décider qu'en français tel adverbe est ou non adverbe de phrase, nous avons dès lors utilisé le critère de Labelle $(1974: 26)$ : l'antéposition à la phrase niée. Est donc considéré comme adverbe de phrase (sous $\mathrm{P}$ ) tout adverbe de phrase pouvant précéder une phrase niée. Ce critère n'a posé de problème que pour très peu d'adverbes du corpus. Ces adverbes sont : parfaitement (truly) ; absolument (awfully/positively) ; indéniablement.

Les trois adverbes ci-dessus étant " affirmatifs ", ils acceptent mal d'être antéposés à une phrase niée. Ex. :

a) Parfaitement, je viendrai.

b) ? Parfaitement, je ne viendrai pas.

a) Absolument, c'est comme ça.

b) ? Absolument, ce n'est pas comme ça.

a) Indéniablement, il a commis le crime.

b) ? Indéniablement, il n'a pas commis le crime.

Le critère de l'antéposition à la phrase niée indique que l'adverbe certainement n'est pas un adverbe de phrase. Ex. :

a) ${ }^{*}$ Certainement, Jean viendra.

b) *Certainement, Jean ne viendra pas. 
Or, l'adverbe certainement est un adverbe de phrase et plus précisément un adverbe de phrase sous $\overline{\mathrm{P}}$. Ex. : Certainement que Jean viendra (ne viendra pas).

L'on pourrait penser qu'en français un adverbe de phrase est soit adverbe sous $P$, soit adverbe sous $\overline{\mathbf{P}}$. En d'autres termes, le fait pour un adverbe de phrase d'être acceptable sous $\overline{\mathbf{P}}$ exclurait la possibilité pour le même adverbe d'être acceptable sous $\mathbf{P}$ (ou l'inverse). Or, ce n'est pas le cas. Ex. : Heureusement, Pierre viendra (ne viendra pas) (Adv. sous P). Heureusement que Pierre viendra (ne viendra pas) (Adv. sous $\overline{\mathbf{P}}$ ).

L'opposition entre les exemples ci-dessus montre que le critère d'antéposition à un phrase niée (par la négation pas) peut exclure à tort certains adverbes de phrase. Pour les rares adverbes pour lesquels ce premier critère pose un problème, nous proposons un deuxième critère : la possibilité pour l'adverbe de répondre à une question globale (c'està-dire une question à laquelle on peut répondre par oui ou non). E. Piquette (1976:184) utilise d'ailleurs déjà ce critère pour déterminer le degré d'autonomie des adverbes par rapport au verbe. Toutefois, l'auteur ne précise pas en quoi ce critère est valable. En fait, l'explication est simple : l'adverbe de phrase ayant pour argument la phrase entière (ou selon une terminologie traditionnelle "modifiant " la phrase entière), il est logique de s'attendre à ce qu'il puisse servir de réponse à une question globale, c'est-à-dire à une question qui, elle aussi, porte sur la phrase entière, et non sur un constituant seulement.

Nos trois " adverbes-problème " et l'adverbe certainement peuvent servir de réponse à une question globale. Ex. : Viendrez-vous ?

a) parfaitement ${ }^{15}$

b) absolument

c) indéniablement ${ }^{16}$

d) certainement.

En résumé, pour le français nous considérons comme adverbe de phrase (sous $\mathbf{P}$ )

- tout adverbe pouvant être antéposé à une phrase niée par pas

- tout adverbe pouvant répondre à une question globale.

Ces deux critères s'appliquent dans l'ordre indiqué.

Pour l'anglais, nous considérons comme adverbe de phrase tout adverbe répertorié comme tel par K. Connors (1973). En effet, la définition étroite de l'adverbe de phrase n'empêche pas la classification des adverbes du corpus d'être exacte.

\subsubsection{L'adverbe de domaine}

L'adverbe de domaine tel que décrit par K. Connors (1973: 29 sq.) correspond aux adverbes de point de vue et $\grave{a}$ un certain nombre d'autres adverbes, notamment des adverbes de sens restrictif (only/seulement).

Les adverbes de point de vue sont en quelque sorte "restrictifs " puisqu'ils indiquent dans quelles limites le locuteur envisage l'énoncé. À un point de vue peut être opposé un autre point de vue, si bien que les adverbes de ce type ont un "contraire " qui vient presque automatiquement à l'esprit. Ex. : Théoriquement (d'un point de vue théorique), c'est réalisable.

L'implication de cet exemple est que pratiquement (en pratique), ce n'est pas réalisable. L'adverbe pratiquement serait donc le "contraire " de théoriquement, au sens où nous l'entendons.

En élargissant un peu la " définition " des adverbes de point de vue, on pourrait, en principe admettre des adverbes comme : localement (par opposition à régionalement ou nationalement), seulement ( $\mathrm{j}$ 'ai seulement dit que..., par opposition à tout ce que j'aurais pu dire), etc.

Élargir de cette façon la définition des adverbes de point de vue semble toutefois impossible pour deux raisons : 
- Les adverbes de domaine comprennent des adverbes n'ayant pas ce " sens restrictif ", par exemple : exactly, exactement ${ }^{17}$; naturally, naturellement ${ }^{18}$; widely, largement/radicalement ; precisely, précisément ${ }^{19}$; respectively, respectivement, etc.

- Si l'on accepte l'analyse de K. Connors (1973), les adverbes de domaine sont un sous-groupe d'une catégorie plus vaste : "extent " et sont donc des adverbes de SV. En anglais, comme en français, certains adverbes ne peuvent se trouver que dans le SV. En anglais, il s'agit des adverbes de domaine et des intensificateurs. En français, il s'agit des intensificateurs et des adverbes de manière. Ceci revient à dire que l'adverbe de domaine est un adverbe de $S V$, alors que l'adverbe de point de vue est un adverbe de phrase.

Il y aurait donc lieu d'effectuer une recherche supplémentaire, car ce problème met en évidence le fait que certains adverbes en -ment ayant un sens restrictif échappent aux catégories adverbiales de Labelle (1973) ${ }^{19}$. Pour ce qui est de notre propre recherche, nous considérerons qu'il n'y a correspondance fonctionnelle que si l'adverbe de domaine (appellation que nous conservons pour l'anglais) correspond à un adverbe de point de vue.

\subsection{Positions syntaxiques des adverbes en -ly}

Voici le tableau proposé par K. Connors (1973 : 39). Les chiffres figurant à gauche des catégories adverbiales indiquent l'interprétation par ordre décroissant $(1=$ interprétation préférentielle).

\begin{tabular}{|c|c|c|c|}
\hline 1 & 2 & 3 & $\begin{array}{l}\text { Adv. } \\
\text { Part. } \\
\mathbf{4}\end{array}$ \\
\hline 1 Adv de phr & 1 temps & $\begin{array}{l}1 \text { extent (inten- } \\
\text { sif de domaine) }\end{array}$ & 1 manière \\
\hline 2 temps & $2 \mathrm{adv}$ de $\mathrm{phr}$ & 2 manière & $\begin{array}{l}2 \text { temps/adv } \\
\text { de phr }\end{array}$ \\
\hline
\end{tabular}

Ainsi qu'on peut le voir d'après le tableau, l'auteur prévoit pour chaque fonction adverbiale une position syntaxique préférentielle, ce qui permet une interprétation automatique "probabiliste".

Comme nous l'avons vu, le français présente les différences suivantes :

- l'adverbe de manière est un adverbe de $S V$; il est donc exclu des positions 1,2 et 4 ;

- l'adverbe du point de vue (domaine) est un adverbe de phrase ; il est donc exclu de la position 3 , mais peut occuper les autres positions;

- les adverbes de phrase peuvent occuper la position 5;

- le français possède des adverbes de phrase sous $P$.

Le tableau devient donc pour le français :

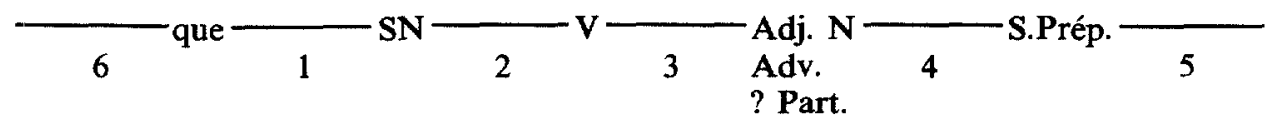

E. Piquette (1976 : $56 \mathrm{sq}$.) montre que la position 2 est disponible pour les adverbes, contrairement à ce qui est généralement avancé. 


\subsection{Divergences fonctionnelles et problèmes de traduction}

La comparaison entre adverbe en -ly et adverbe en -ment est compliquée par le fait qu'à un adverbe en -ly correspondent généralement plusieurs adverbes en -ment. Par exemple, cinq adverbes en -ment correspondent à l'adverbe fairly (voir ci-dessous), huit à l'adverbe positively. Nous donnons ci-dessous les adverbes en -ment correspondant à l'adverbe fairly, avec indication des fonctions respectives.

Fairly = manière ou intensificateur : équitablement, impartialement, honnêtement, loyalement $=$ manière $;$ moyennement $=$ manière et intensificateur $;$ assez $=$ intensificateur.

Étant donné cette situation, un des problèmes de la traduction consiste à choisir l'adverbe en -ment requis par le texte de départ ; en fait, ce choix dépend d'abord de la fonction de l'adverbe de départ. Ainsi, si l'adverbe fairly est utilisé comme intensificateur, les seuls adverbes possibles (parce qu'ils ont la même fonction) sont moyennement et assez. Le choix entre deux ou plusieurs adverbes dépendra ensuite, s'il y a lieu :

- des restrictions de cooccurrence; ce serait le cas si l'adverbe fairly avait la fonction manière, car cinq adverbes en -ment deviennent alors disponibles;

- du souci d'éviter toute ambiguïté (dans le cas d'adverbes ayant plusieurs fonctions et plus particulièrement d'adverbes pouvant être à la fois adverbe de phrase et adverbe de SV);

- de considérations stylistiques.

Des exemples d'ambiguitté ont été donnés en 1.4 et il a été montré que l'ambiguïté peut être résolue soit par l'utilisation d'un syntagme prépositionnel, soit par le recours à un intensificateur (dans le cas d'un adverbe occupant une position syntaxique ambiguë et dont une des fonctions est manière).

Des considérations stylistiques feraient rejeter l'exemple $a$ en faveur de $b$ :

a) Est-ce vraiment vrai?

a) Est-ce bien vrai?

Les divergences fonctionnelles est un autre problème auquel le traducteur (humain ou non) doit faire face. Par divergence fonctionnelle, nous entendons le cas où l'adverbe en -ment n'a pas la fonction exigée par l'adverbe en -ly ou l'inverse. Nous donnons ci-dessous des exemples de divergence fonctionnelle pour chacune des fonctions adverbiales. Le signe [-] indique une fonction inexistante; dans ce cas, une traduction est proposée. Le signe [+] indique que l'adverbe en -ment a une fonction que n'a pas l'adverbe en $-l y$.

relatively

domaine

surprisingly

adv. de phrase

remarkably

adv. de phrase relativement

- point de vue (domaine)

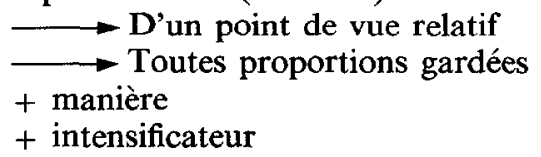

*surprenamment

$\longrightarrow$ Chose surprenante...

Il est surprenant que...

remarquablement

- adv. de phrase

Chose remarquable...

Il est remarquable que... 
$\begin{array}{ll}\text { easily } & \text { aisément, tranquillement } \\ \text { intensificateur } & \text { manière }\end{array}$

a) He is easily the best (Robert Collins)

b) Il est de loin (sans aucun doute) le meilleur.

\section{ultimately finalement \\ temps [+ futur] temps [- futur]}

a) He will ultimately build a block of flats here. (Robert Collins)

b) Nous envisageons de construire un immeuble ici par la suite (à l'avenir).

$\begin{array}{ll}\text { typically } & \text { typiquement } \\ \text { temps } & \text { - temps } \\ & - \text { manière }\end{array}$

a) It was typically wet that day. (Robert Collins)

b) Il pleuvait beaucoup ce jour-là, comme d'habitude.

Les quelques exemples ci-dessus montrent clairement que les fonctions adverbiales ne sont pas les mêmes d'une langue à l'autre et que la traduction est tributaire de la fonction. L'absence d'une fonction particulière oblige à utiliser autre chose qu'un adverbe :

w syntagme prépositionnel (d'un point de vue relatif);

II syntagme nominal + adjectif (chose étonnante), pour autant que le français ait l'adjectif correspondant à l'adverbe en $-l y$;

une « phrase » : Il est surprenant que... Cette traduction n'est de nouveau possible que pour autant que le français ait l'adjectif correspondant à l'adverbe en -ly. Ce n'est pas toujours le cas. Ex. :

a) Hopefully they will come.

b) Espérons qu'ils viendront.

Dans la plupart des cas, lorsqu'il y a divergence fonctionnelle, la traduction est une paraphrase de la fonction de l'adverbe de départ. Il serait donc intéressant d'établir une liste des fonctions et des différentes paraphrases possibles des adverbes en -ment. Pareille liste mettrait notamment en évidence les deux phénomènes suivants ${ }^{20}$ :

- Un syntagme prépositionnel donné peut ne correspondre qu'à une des fonctions de l'adverbe considéré. Par exemple l'adverbe généralement correspond à : en général; de façon générale. En fait, en général correspond à la fonction temps de l'adverbe, tandis que de façon générale correspond à la fonction point de vue du même adverbe.

- Certains syntagmes prépositionnels n'ont pas le même sens que l'adverbe auquel ces syntagmes ressemblent. Par exemple, brièvement et en bref. Brièvement signifie peu de temps ou en peu de mots. Ex. :

Il est venu brièvement (peu de temps)

Il l'a expliqué brièvement (en peu de mots)

En bref a le sens de somme toute et joue le rôle d'un adverbe de phrase, fonction que l'adverbe brièvement n'a pas. Ex. :

a) *Brièvement, il est venu

b) Brièvement, il n'est pas venu

c) En bref, il est (n'est pas) venu.

Donc le syntagme prépositionnel comble des lacunes dans les fonctions des adverbes en -ment (en ces sens, les S.Prép. sont dès lors plus « mobiles" que les adverbes). I1 en est de même de certains syntagmes nominaux. Ex. : 
a) *Étonnamment, Pierre est venu.

b) *Étonnamment, Pierre n'est pas venu.

c) Chose étonnante, Pierre est venu.

d) Chose étonnante, Pierre n'est pas venu.

Ainsi que nous l'avons vu, à un adverbe en -ly correspondent plusieurs adverbes en -ment. Certains adverbes en -ment ont une ou des fonctions qui n'a pas d'adverbe en -ly. Ces fonctions sont indiquées au moyen d'un [+] et nous avons attribué l'étiquette "fonction supplémentaire ". En fait il ne s'agit pas de fonctions supplémentaires à proprement parler. En effet, selon K. Connors (1973 : 32), en anglais le nombre de fonctions pour une même forme adverbiale est de trois. Ex. :

manière $\quad$ a) He acted naturally = "in a natural manner"

extension $\quad$ b) He was naturally nervous = "by nature»

adv. de phr. c) Naturally, he was nervous = "It was natural that".

Labelle (1974 : 14) établit une distinction nette entre adverbe de phrase et adverbe de SV. D'autre part, il affirme qu'il est possible de distinguer, dans le groupe SV, les adverbes de manière des adverbes d'intensité (Labelle $1974: 26$ ). Tout ceci implique qu'en français deux fonctions seulement seraient possibles au sein d'une même forme adverbiale : adv. de SV : manière ou intensité ; adv. de phrase.

En pratique, la distinction manière/intensité n'est pas claire. Il se pourrait donc que le français ait, lui aussi, trois fonctions possibles pour une même forme adverbiale : manière ; intensité ; adv. de phrase.

Le nombre maximal de fonctions serait dès lors identique en français et en anglais.

Notre comparaison des catégories sémantiques ainsi que les positions syntaxiques des adverbes en -ly et des adverbes en -ment, nous a permis de constater :

- Que les adverbes de SV ne sont pas les mêmes dans les deux langues (anglais, domaine + intensité / français, manière + intensité). Ceci pose certains problèmes, notamment en ce qui concerne les adverbes ayant, en français, un sens restrictif.

- Que le français a plus de positions adverbiales (6) que l'anglais (4) ; le français, contrairement à l'anglais, a notamment des adverbes sous $P$. Toutefois, le nombre maximal de fonctions serait de trois en anglais et de deux en français.

- Que les adverbes différents d'une langue à l'autre, non seulement en ce qui concerne les fonctions, mais même en ce qui concerne des "traits» secondaires comme [+ futur]. - Que le syntagme prépositionnel, en français, joue plusieurs rôles : il comble les lacunes de la dérivation en -ment tant au niveau morphologique qu'au niveau fonctionnel ; il désambiguïse.

\section{CONCLUSION}

L'ensemble de notre recherche avait pour objectifs

- de déterminer pourquoi il y a beaucoup plus d'adverbes en -ly que d'adverbes en -ment. Certaines hypothèses ont été proposées à la section 1.

- de clarifier le rôle du syntagme prépositionnel en français. Nous avons tenté, à la section 2, de préciser le rôle du S.Prép.

Au cours de notre recherche, nous avons également esssayé de tenir compte de certains problèmes de traduction, ce qui nous a obligé à comparer le système adverbial de l'anglais à celui du français. Nous sommes conscients de ce que cette tentative a de superficiel et de fragmentaire. Toutefois, nous espérons que ce premier essai incitera d'autres chercheurs à examiner les problèmes de traduction sous l'angle «fonctionnel ".

CHANTAL BERTRAND, UNIVERSITÉ DE MONTREAL 
Notes

Nous remercions vivement Monsieur A. Clas d'avoir, avec son efficacité habituelle, accepté de réviser le texte de notre article.

1. Il s'agit des 322 adverbes en -ly les plus fréquents parmi les 3000 mots les plus utilisés de l'anglais (annexe IV).

2. Contrairement aux autres adverbes en -ment, les intensificateurs (aussi appelés adverbes de degré) peuvent être également formés à partir de noms : diable diablement, vache vachement ; d'interjections : bigre $\longrightarrow$ bigrement, bougre $\longrightarrow$ bougrement, diantre $\longrightarrow$ diantrement, fichtre $\longrightarrow$ fichtrement.

Dans le cadre de la théorie d'Aronoff (1976), ces adverbes seraient entrés au lexique, leur sens n'étant pas prévisible.

D'autre part, selon A. Dauzat (1947 : 339-341), historiquement, les adverbes en -ment ont été formés sur le féminin de l'adjectif. Quand l'adjectif masculin se terminait par une voyelle, l'e du féminin a généralement disparu (aisée aiséement aisément; due duement dûment). Dans de rares cas, il y a deux formes (gaiement et gaîment).

La théorie morphologique actuelle semble plutôt prôner une base masculine pour les adverbes en -ment. Nous n'entrerons pas ici dans ce débat.

3. Or, ce dictionnaire comprend des adverbes à notre avis inexistants (mais possibles au sens morphologique du terme : antécédemment, opulemment), rares ou littéraires (vigilamment, semblablement, impertinemment), vieillis ou appartenant à la langue classique (quellement, bellement).

4. Si l'adjectif n'est pas un déverbal (ce qui au point de vue orthographique se manifeste par une finale en -ent et non plus en -ant), l'adverbe est possible (éminent, éminemment).

5. La situation est en réalité plus complexe que cela, car on n'a pas non plus *gourmamment (de façon gourmande), or il ne s'agit pas d'un déverbal.

6. La seule exception que nous ayons relevée est l'adverbe étonnamment

7. Nous insistons sur le mot productif. Le français écrit connaît en effet quelques adverbes de ce type : achetez français, voir clair. Il s'agit toutefois d'un petit groupe de formes figées.

8. Ceci est noté par Labelle (1974: 33-34), mais, à notre avis, de façon inutilement détaillée. Il s'agit de qualités perceptibles par les sens, que ces qualités soient attribuées à des objets ou à des personnes.

9. noirement figure dans le DIF, mais ne figure ni dans le Petit Robert ni dans le Larousse encyclopédique.

10. C"est le cas de bellement qui figure dans le DIF, mais qui est indiqué comme vieux par le Petit Robert (PR); laidement qui figure dans le DIF et au PR dans les sens suivants : avec laideur : tableau laidement encadré (cette phrase me paraît impossible. Il me semble qu'actuellement on dirait un tableau mal encadré) ; rare : avec bassesse, malhonnêteté, voir ignoblement : Il s'est comporté laidement (cette phrase me paraît également impossible ; il me semble qu'actuellement on emploierait ignoblement) ; grandement dans certains dialectes. Cet adverbe semble, par contre, s'être maintenu au Québec et figure au Petit Robert; joliment existe comme adverbe de manière et comme intensificateur.

11. Nous ne considérons que le préfixe négation in-parce qu'en français, c'est de loin le préfixe négatif par excellence.

12. Certaines paires d'adverbes présentent en effet une variation de sens et non une opposition de sens : conséquemment ( $=$ en conséquence) ; inconséquemment ( $=$ avec inconséquence); différemment ( $=$ de façon différente); indifféremment $(=$ avec indifférence); dépendamment (Québec $=$ selon); indépendamment ( $=$ avec indépendance).

Dans le cadre de la théorie d'Aronoff (1976), ces adverbes seraient entrés au lexique puisque leur sens n'est pas prévisible à partir de la base et des affixes.

13. Dans la plupart des cas, l'adverbe positif ne peut pas constituer une base étant donné qu'il ne s'agit pas d'un mot au sens d'Aronoff (1976)

Selon M. Picard (1982 : 191), la situation est la même en anglais (inept/*ept; innocent/"nocent ; inert/

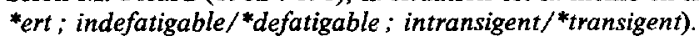

14. Exemple de Labelle (1974: 15) qui choisit de ne pas tenir compte des pauses. Pour une discussion détaillée des pauses, voir E. Piquette (1976: $50 \mathrm{sq}$.). Les points suivants concernent particulièrement notre sujet : la position 1 est généralement suivie d'une pause, sans que cette pause corresponde à une mise en relief particulière; presque tous les adverbes peuvent occuper, sans pause, les positions 3 , 4 et 5 .

15. L'adverbe parfaitement nous paraît vieilli en tant qu'adverbe de phrase.

16. L'adverbe indéniablement nous paraît également vieilli en tant qu'adverbe de phrase. À notre avis, actuellement, on emploierait plutôt l'adverbe indiscutablement.

17. Les adverbes exactement, naturellement et précisément sont des adverbes de phrase (modaux) dans la terminologie de Labelle.

18. La catégorie lieu n'existe pas non plus. Or, un des adverbes du corpus, locally peut avoir cette interprétation (Localement, on manque de vivres). 
19. Cette liste pourrait également indiquer la préférence des locuteurs pour l'adverbe (pris isolément) ou pour le S.Prép. La préférence du locteur pour le S.Prép. indiquerait que l'adverbe est peu utilisé, en tout cas dans le dialecte du locuteur en question.

\section{ANNEXE 1 \\ Adverbes : fonctions impossibles en français}

\section{A. ADVERBES EN -MENT NE POUVANT ÊTRE ADV. DE POINT DE VUE}

\author{
exactly \\ relatively (speaking) \\ naturally \\ widely \\ precisely \\ respectively \\ chiefly \\ consciously \\ reasonably \\ proportionately \\ characteristically
}

Adverbes n'ayant pas un sens restrictif

\section{Adverbes ayant un sens restrictif}

only

personally

publicly

physically

solely

comparatively

locally

locally
properly en tant qu'adverbe de point de vue correspond au frant

\author{
exactement \\ relativement (*parlant) \\ naturellement \\ largement, radicalement \\ précisément \\ respectivement \\ principalement (surtout) \\ consciemment \\ raisonnablement \\ proportionnellement \\ typiquement ( $\rightarrow$ "chose typique, ... ")
}

\author{
seulement \\ personnellement \\ publiquement \\ physiquement \\ seulement, uniquement \\ comparativement (à), relativement (à)
}

en tant qu'adverbe de point de vue correspond au français $\grave{a}$ proprement parler 


comfortably
dimly
distinctly
unexpectedly
duly
gentuinely
solidly
heartily
fatly

confortablement, agréablement, à l'aise

faiblement, indistinctement, vaguement, imparfaitement

distinctement, clairement (sans équivoque), expressément, formellement

subitement, inopinément

đûment

authentiquement, véritablement, sincèrement

solidement, massivement

chaleureusement

catégoriquement, absolument

D. ADVERBES EN -MENT NE POUVANT ÊTRE ADVERBE DE MANIÈRE

highly

terribly

historically hautement (Petit Robert vieilli comme adv. de manière)

terriblement, drôlement, rudement, affreusement, horriblement (Littré : vieilli comme adverbe de manière. Ex. : Il tonnait terriblement $=$ de façon terrible.)

historiquement

E. ADVERBES EN -MENT NE POUVANT ETTRE ADVERBE DE TEMPS (OU DONT UN TRAIT CHANGE)

ultimately [forward-looking]

finalement [backward-looking]

Ex. : We will ultimately build a block of flats here $\Leftrightarrow$ par la suite)

typically

Ex. It was typically wet that day

typiquement

Il pleuvait beaucoup ce jour-là, comme d'habitude.

ANNEXE II

Adverbes : fonctions supplémentaires du français

$\begin{array}{ll}\begin{array}{l}\text { ANGLAIS } \\ \text { Adverbe } \\ \text { completely }\end{array} & \begin{array}{l}\text { Fonction(s) } \\ \text { intensif. }\end{array} \\ \text { exactly } & \begin{array}{l}\text { manière } \\ \text { domaine }\end{array} \\ \text { fully } & \text { intensif. } \\ \text { approximately } & \text { domaine } \\ \text { primarily } & \text { domaine }\end{array}$

\section{FRANÇAIS}

Adverbe

complètement

exactement

précisément

entièrement

complètement

approximativement

essentiellement

principalement

primitivement

adv. de phrase vraiment

réellement

franchement
Fonction(s)

intensif.

+ manière

manière

- point de vue

+ modal

intensif.

+ manière

? point de vue

+ manière

$?$ point de vue

+ intensif.

? point de vue

? temps

+ temps

- point de vue

modal

? intensif.

idem

modal

+ manière 


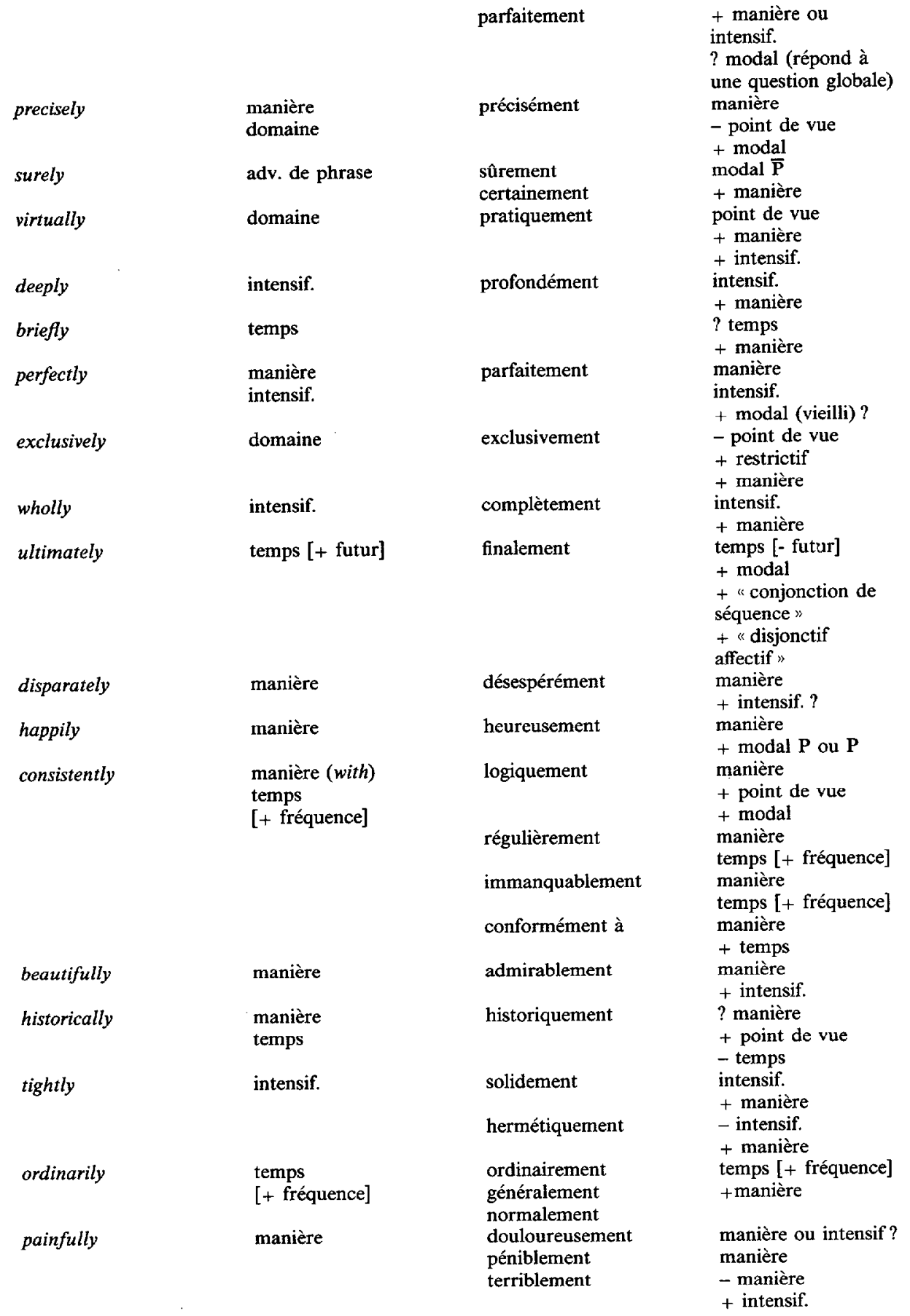




\begin{tabular}{|c|c|c|c|}
\hline squarely & manière & $\begin{array}{l}\text { carrément } \\
\text { honnêtement }\end{array}$ & $\begin{array}{l}\text { - manière } \\
\text { + intensif. } \\
\text { manière }\end{array}$ \\
\hline locally & domaine & localement & $\begin{array}{l}\text { - point de vue } \\
\text { (+ restrictif) } \\
+ \text { manière } \\
+ \text { modal (lieu?) }\end{array}$ \\
\hline unusually & manière & $\begin{array}{l}\text { exceptionnellement } \\
\text { extraordinairement }\end{array}$ & $\begin{array}{l}\text { manière } \\
+ \text { intensif. } \\
\text { - manière } \\
+ \text { intensif. }\end{array}$ \\
\hline wonderfully & manière & $\begin{array}{l}\text { merveilleusement } \\
\text { admirablement }\end{array}$ & $\begin{array}{l}\text { manière } \\
+ \text { intensif. }\end{array}$ \\
\hline awfully & intensif. & $\begin{array}{l}\text { terriblement } \\
\text { vraiment } \\
\text { absolument }\end{array}$ & $\begin{array}{l}\text { intensif. } \\
\text { intensif. } \\
\text { intensif. } \\
+ \text { manière } \\
? \text { modal (répond à } \\
\text { une question globale) }\end{array}$ \\
\hline genuinely & intensif. & $\begin{array}{l}\text { authentiquement } \\
\text { véritablement } \\
\text { sincèrement }\end{array}$ & $\begin{array}{l}\text { - intensif. } \\
\text { +manière } \\
\text { intensif. } \\
\text { intensif. } \\
\text { +modal } \\
\text { +manière }\end{array}$ \\
\hline positively & $\begin{array}{l}\text { manière } \\
\text { adv. de phrase }\end{array}$ & indéniablement & $\begin{array}{l}\text { intensif. } \\
\text { ? modal (répond à } \\
\text { une question globale) }\end{array}$ \\
\hline superbly & manière & $\begin{array}{l}\text { superbement } \\
\text { magnifiquement }\end{array}$ & $\begin{array}{l}\text { manière } \\
+ \text { intensif. }\end{array}$ \\
\hline unhappily & manière & malheureusment & $\begin{array}{l}\text { - manière } \\
+ \text { modal }\end{array}$ \\
\hline conspicuously & manière & visiblement & $\begin{array}{l}\text { manière } \\
+ \text { modal }\end{array}$ \\
\hline & & manifestement & $\begin{array}{l}\text { - manière } \\
+ \text { modal }\end{array}$ \\
\hline experimentally & manière & expérimentalement & $\begin{array}{l}\text { manière } \\
+ \text { point de vue }\end{array}$ \\
\hline financially & manière & financièrement & $\begin{array}{l}\text { manière } \\
+ \text { point de vue }\end{array}$ \\
\hline magnificently & manière & magnifiquement & $\begin{array}{l}\text { manière } \\
+ \text { intensif. }\end{array}$ \\
\hline sincerely & manière & sincèrement & manière \\
\hline
\end{tabular}

\section{ANNEXE II (suite) \\ Adverbes : fonctions supplémentaires - Exemples}

complètement : manière

exactement, précisément : + modal

entièrement, complètement : + manière approximativement : + manière

$$
+ \text { point de vue }
$$

essentiellement : + intensif.

principalement

primitivement : + temps
Lire un livre complètement.

? Exactement (précisément), il l'a fait

? Exactement (précisément), il ne l'a pas fait

Lire un livre entièrement (complètement).

a) Évaluer une cuvre d'art approximativement.

d) Approximativement, je dirais que cela fait $5 \%$ $=$ D'un point de vue approximatif

L'homme et la femme sont essentiellement différents.

Elle en voulait principalement à son mari (= avant

tout)

Primitivement, l'homme vivait dans des cavernes (= à l'origine) 
a) vraiment : + intensif

b) réellement : + intensif.

franchement : + manière

a) parfaitement : + manière ou intensif.

b) parfaitement : modal ?

\author{
précisément : + modal \\ sûrement, certainement : + modal \\ pratiquement $:+$ manière \\ + intensif. \\ profondément : + manière \\ brièvement : ? temps \\ exclusivement : adv. " restrictif "
}

+ manière

finalement : temps [- futur ]
Est-il vraiment coupable?

Est-il réellement coupable ?

Il l'a dit bien (très) franchement.

Un tableau parfaitement réussi.

Répond à une question globale, mais me semble vieilli dans cet emploi.

- Vous n'allez pas partir par ce temps?

- Parfaitement

Précisément, il va (ne va pas) le faire.

Sûrement (certainement) que Pierre viendra

(ne viendra pas).

a) Cet appareil est très pratiquement conçu

b) Il est pratiquement ( = virtuellement) incapable de se déplacer (emploi critiqué, selon le Petit Robert).

Il a creusé profondément.

a) Il est venu brièvement (peu de temps ou de façon brève ?).

b) Il l'a expliqué brièvement.

a) Il lit exclusivement des ouvrages philosophiques.

b) Du mois de janvier au mois d'août exclusivement.-

c) Gide : " Le dilettante qui comprend tout, précisément parce qu'il n'aime rien passionnément, c'est-à-dire exclusivement. " (Petit Robert)

a) *Nous envisageons de construire un immeuble ici finalement (ultimately).

b) Nous envisageons de construire un immeuble ici par la suite.

c) Finalement, Jean est venu (n'est pas venu).

Est aussi "conjonctif de séquence " (introduisant une énumération d'au moins trois êléments) et "disjonctif affectif ", ainsi que le fait remarquer E. Piquette (1976: 188).

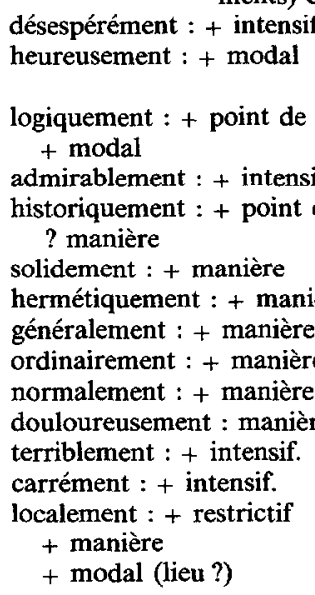

exceptionnellement, anormalement extraordinairement : + intensif merveilleusement, admirablement : + intensif.

absolument : + manière ?modal

authentiquement : + manière
Il est désespérément borné.

a) Heureusement que Pierre est venu

b) Heureusement, Pierre est (n'est pas) venu.

a) Logiquement ce raisonnement est faux.

b) Logiquement, Pierre viendra (ne viendra pas).

Ce tableau est admirablement réussi.

a) Un fait historiquement explicable.

Une maison solidement construite.

Une bouteille fermée hermétiquement.

C'est lui qui a traité le sujet le plus généralement.

S'habiller (parler) (très) ordinairement.

Parler normalement ( $\sim$ anormalement).

Être douloureusement blessé.

Je suis tellement vexée.

Cela est carrément ( $=$ absolument) faux.

a) Localement, on pense que ce n'est pas faisable.

b) Etre endormi localement.

c) Localement, on manque de vivres. Localement, on ne manque pas de vivres.

Être exceptionnellement (anormalement,

extraordinairement) doué.

Être merveilleusement (admirablement)

doué.

a) Verbe employé absolument.

b) Répond à une question globale

- C'est bien cela ?

- Absolument!

Ces meubles sont authentiquement anciens.

(Larousse des débutants) b) ?Cela s'est déroulé historiquement. 
sincèrement : + modal

indéniablement : + modal ?

formellement, catégoriquement :

+ intensif

positivement : + intensif

superbement, magnifiquement :

+ intensif.

malheureusement : + modal

visiblement : + modal

manifestement : +modal

expérimentalement $:+$ point de vue

financièrement : + point de vue
Sincèrement, je crois que Pierre viendra

(ne viendra pas)

a) Ce crime il l'a indéniablement (indiscutablement)

commis. Paraphrase : il est indéniable que...

b) A-t-il commis ce crime? Indéniablement.

N.B. Cet adverbe me semble "vieilli " dans cet emploi.

Il nous a formellement (catégoriquement)

défendu de le faire.

Il nous fut positivement impossible de nous suppor-

ter.

a) Ce travail est magnifiquement réussi.

b) Ce travail est superbement réussi.

Malheureusement, il viendra (ne viendra pas)

a) Visiblement, il viendra.

b) Manifestement, il ne viendra pas.

Expérimentalement, c'est irréalisable.

Financièrement, c'est impossible.

N.B. Un seul adverbe en $-l y$ semble avoir plus de fonctions que l'adverbe en -ment correspondant : reasonably: raisonnablement :

manière, adv. de phrase,

manière, - adv. de phrase,

intensificateur

- intensificateur

\section{ANNEXE III \\ Adverbes en -ly ne correspondant à aucun adverbe en -ment}

$\begin{array}{ll}\begin{array}{l}\text { hardly } \\ \text { highly }\end{array} & \text { à peine, guère } \\ \text { largely } & \text { fort } \\ & \text { en grande partie, dans une large mesure surtout, pour la plupart, en } \\ \text { similarly } & \text { général } \\ \text { duccessfully } & \text { avec succès } \\ \text { scarcely } & \text { à peine, avec soin, de façon soignée à maintes reprises, très souvent. } \\ & \text { N.B. L'adj. répétitif existe. } \\ \text { significantly } & \text { de façon significative } \\ \text { emotionally } & \text { avec émotion } \\ \text { permanently } & \text { de façon permanente } \\ \text { safely } & \text { sans accident, sans risque, sans danger } \\ \text { satisfactorily } & \text { d'une manière satisfaisante (acceptable) } \\ \text { conceivebly } & \text { de façon concevable } \\ \text { reportedly } & \text { selon un rapport, selon des "on dit " } \\ \text { vividly } & \text { d'une manière vivante (colorée) } \\ \text { hopefully } & \text { avec espoir (optimisme), de façon encourageante (adv de phrase : } \\ \text { importantly } & \text { "Espérons que... ") } \\ \text { prominently } & \text { d'un air important (adv. de phrase : " chose importante... ") } \\ \text { conveniently } & \text { bien en vue } \\ \text { excitedly } & \text { d'une manière commode, sans inconvénient } \\ \text { hopelessly } & \text { avec agitation, d'une manière agitée } \\ \text { averwhelmingly } & \text { sans espoir } \\ \text { reluctantly } & \text { d'une manière écrasante (accablante) } \\ & \text { à regret, à contrecour, sans enthousiasme } \\ & \end{array}$

Adverbes en -ly dont certaines traductions seulement correspondent à un adverbe en -ment

slightly

greatly

badly

barely

loudly

thoughfully

légèrement, un peu

grandement, grand (Il est grand temps)

grièvement, gravement, sérieusement, mal

pauvrement, à peine, tout juste, sans détails

bruyamment, vigoureusement, fort

pensivement, avec prévenance 
duly

wearily dûment, comme il faut (convient), en temps voulu (utile) en effet, bien entendu

péniblement, d'un ton las (fatigué)

\section{ANNEXE IV}

K. Connors (1973) : "English adverb functions : their distribution and relations ».

\section{CORPUS}

Manner

simply

suddenly

directly

clearly

obviously

slowly

easily

highly

exactly

quickly

carefully

naturally

rapidly

equally

heavily

fairly

truly

properly
Non-Manner

only : extent (domain)

early : time (non-durative)

really : $S$ - $a d v$

probably : S-adv

usually:time ( + frequency)

finally : time (non-durative)

simply : S-adv, extent (domain)

actually : S-adv

especially : extent (domain)

certainly : S-adv

nearly : extent (degree)

merely : extent (domain)

generally : time ( + frequency)

clearly : S-adv

apparently : S-adv

immediately : time (forward-

looking)

recently : time (backward-

looking)

daily : time (+ frequency)

obviously : S-adv

completely : extent (degree)

easily : extent (degree)

hardly : extent (degree)

highly : extent (degree)

exactly : extent (domain)

entirely : extent (degree)

frequently : time ( + frequency)

relatively : extent (domain) slightly : extent (degree)

fully : extent (degree)

approximately : extent (do-

main)

naturally : S-adv, extent (domain)

largely : extent (degree)

closely : extent (degree)

primarily : extent (domain)

equally : extent (degree)

greatly : extent (degree)

possibly : S-adv

heavily : extent (degree)

fairly : extent (degree)

previously : time (backward-

looking)

truly : S-adv

properly : extent (domain)

\begin{tabular}{|c|c|}
\hline practically & $\begin{array}{l}\text { practically : extent (domain) } \\
\text { eventually : time (forward- } \\
\text { looking) } \\
\text { widely : extent (domain) }\end{array}$ \\
\hline gradually & $\begin{array}{l}\text { necessarily : S-adv } \\
\text { extremely : extent (degree) }\end{array}$ \\
\hline firmly & $\begin{array}{l}\text { partly : extent (degree) } \\
\text { occasionally : time ( }+ \text { fre- } \\
\text { quency) }\end{array}$ \\
\hline $\begin{array}{l}\text { precisely } \\
\text { quietly }\end{array}$ & $\begin{array}{l}\text { precisely : extent (domain) } \\
\text { essentially : S-adv } \\
\text { surely : S-adv }\end{array}$ \\
\hline seriously & $\begin{array}{l}\text { seriously : extent (degree) } \\
\text { considerably : extent (degree) } \\
\text { mostly : extent (degree) }\end{array}$ \\
\hline readily & $\begin{array}{l}\text { sufficiently : extent (degree) } \\
\text { constantly : time (+ frequency) } \\
\text { rarely : time (+ frequency) } \\
\text { virtually : extent (domain) }\end{array}$ \\
\hline personally & $\begin{array}{l}\text { personally : extent (domain) } \\
\text { presumably : S-adv }\end{array}$ \\
\hline thoroughly & $\begin{array}{l}\text { thoroughly : extent (degree) } \\
\text { deeply : extent (degree) } \\
\text { briefly : time (durative) } \\
\text { inevitably : S-adv }\end{array}$ \\
\hline sharply & $\begin{array}{l}\text { sharply : extent (degree) } \\
\text { simultaneously : time (non- } \\
\text { durative) } \\
\text { specifically : S-adv }\end{array}$ \\
\hline $\begin{array}{l}\text { effectively } \\
\text { strongly } \\
\text { automatically }\end{array}$ & $\begin{array}{l}\text { effectively : S-adv } \\
\text { strongly : extent (degree) }\end{array}$ \\
\hline similarly & $\begin{array}{l}\text { normally : time (+ frequency) } \\
\text { similarly : S-adv } \\
\text { substantially : extent (degree) } \\
\text { presently : time ((Br. Eng.) } \\
\text { forward-looking) } \\
\text { presently : time ((Am.) dura- } \\
\text { tive) }\end{array}$ \\
\hline badly & $\begin{array}{l}\text { badly : extent (degree) } \\
\text { currently : time (durative) }\end{array}$ \\
\hline $\begin{array}{l}\text { openly } \\
\text { reasonably }\end{array}$ & $\begin{array}{l}\text { reasonably : S-adv, extent (de- } \\
\text { gree) }\end{array}$ \\
\hline shortly & $\begin{array}{l}\text { shortly : time (forward- } \\
\text { looking) }\end{array}$ \\
\hline st & $\begin{array}{l}\text { strictly : extent (domain) } \\
\text { unfortunately : } \mathrm{S}-\mathrm{adv}\end{array}$ \\
\hline
\end{tabular}

eventually : time (forward-

looking)

necessarily : S-adv

partly : extent (degree)

casionally : time (+ frequency)

essentially : S-adv

surely : S-adv

mostly : extent (degree)

sufficiently : extent (degree)

(+ frequency)

virtually : extent (domain)

: extent (domain)

mably : S-ad

deeply : extent (degree)

briefly : time (durative)

evitably : S-adv

simultaneously : time (nondurative)

specifically : S-adv

effectively : S-adv

normally : time ( + frequency)

similarly : S-adv

forward-looking)

resently : time (Am.) dura-

badly : extent (degree)

reasonably : S-adv, extent (degree)

looking)

unfortunately : S-adv 


accordingly
gently
lightly
perfectly
softly
successfully
deliberately

publicly

wildly accurately

roughly

originally

desperately

freely

streadily

definitely

happily

consistently

individually

neatly

abruptly

formally accordingly : S-adv

barely : extent (degree)

consequently : S-adv

invariably : time (+ frequency)

lightly : extent (degree)

mainly : extent (domain)

perfectly : extent (degree)

respectively : extent (domain)

puraly : extent (domain)

commonly : time $(+$ frequency)

promptly : time (forward-

looking)

absolutely : extent (degree)

publicly : extent (domain)

utterly : extent (degree)

continually : time (+ fre-

quency)

evidently : S-adv

partially : extent (degree)

wildly : extent (degree)

exclusively : extent (domain)

roughly : extent (domain)

scarcely : extent (degree)

undoubtedly : S-adv

weekly : time (+ frequency)

wholly : extent (degree)

continuously : time $(+$ frequency)

monthly : time (+ frequency)

originally : time (backwardlooking)

ultimately : time (forward-

looking)

chiefly : extent (domain)

regularly : time (+ frequency)

steadily : time (+ frequency)

totally : extent (degree)

definitely : S-adv

basically : extent (domain)

fortunately : S-adv

physically : extent (domain)

solely : extent (domain)

temporarily : time (durative)

consistently : time (+ frequency)

instantly : time (non-durative)

initially : time (backwardlooking)

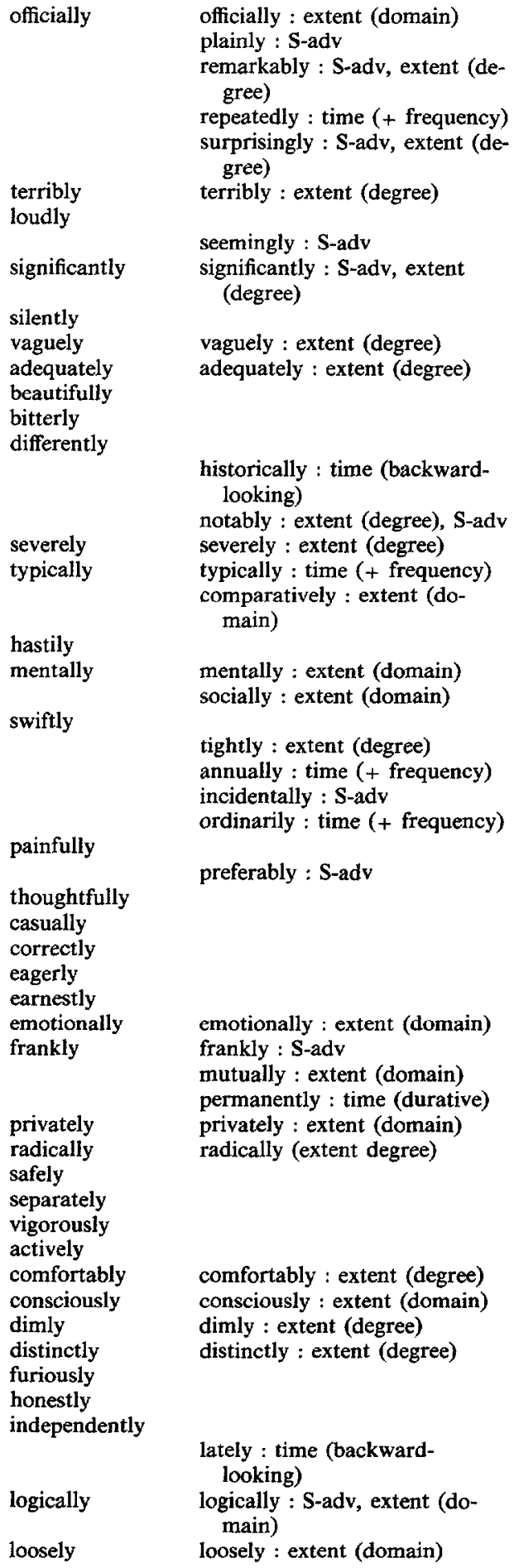

terribly : extent (degree)

seemingly : S-adv

significantly : S-adv, extent

(degree)

vaguely : extent (degree)

adequately : extent (degree)

historically : time (backwardlooking)

notably : extent (degree), S-adv

severely : extent (degree)

typically : time ( + frequency)

comparatively : extent (domain)

mentally : extent (domain)

socially : extent (domain)

tightly : extent (degree)

annually : time (+ frequency)

incidentally : S-adv

ordinarily : time (+ frequency)

preferably : S-adv

emotionally : extent (domain)

frankly : S-adv

mutually : extent (domain) permanently : time (durative) privately : extent (domain) radically (extent degree)

comfortably : extent (degree)

consciously : extent (domain)

dimly : extent (degree)

distinctly : extent (degree)

lately : time (backwardlooking)

logically : S-adv, extent (domain)

loosely : extent (domain) 


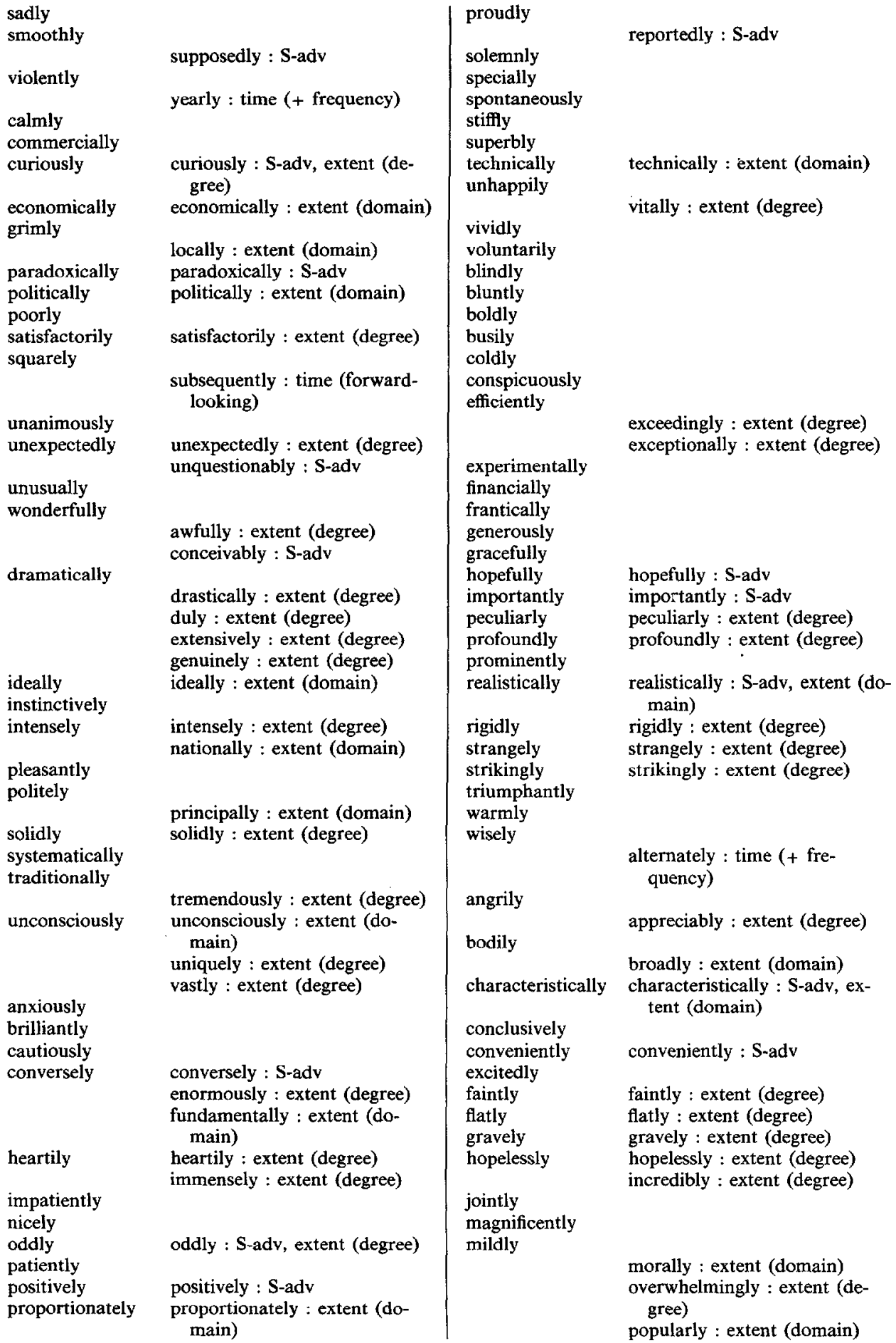


potentially : extent (domain) predominantly : extent (domain)

quarterly : time (+ frequency) reluctantly sincerely

wearily

spiritually : extent (domain)

\section{BIBLIOGRAPHIE}

ARONOFF, M. (1976) : Word Formation in Generative Grammar, Cambridge, Mass., MIT Press.

CONNORS, K. (1973) : "English Adverb Functions : their Distribution and Relations. Études de linguistique appliquée à la traduction automatique ", Université de Montréal, pp. $29-49$.

DAUZAT, A. (1974) : Grammaire raisonnée de la langue française, Lyon, Éditions IAC.

DRAPEAU, L. (1982) : "L'utilisation adverbiale des adjectifs en français populaire", dans la Syntaxe comparée du français standard et populaire : approches formelle et fonctionnelle, C. Lefebvre éditeur, Office de la langue française.

DUBUISSON, C. (1975) : les Transformations radicales et préservatrices de structure en français, thèse de doctorat, Université de Montréal.

Grand Larousse encyclopédique (1960) : Paris, Librairie Larousse, 10 vol.

JACKENDOFF, R. (1972) : Semantic Interpretation in Generative Grammar, Cambridge, Mass., MIT Press. JUILLAND, A. (1965) : Dictionnaire inverse de la langue française, Paris, Mouton.

LABELLE, F. (1974) : Étude syntaxique des adverbes en -ment et de certaines de leurs propriétés syntaxtiques, mémoire de maîtrise, Université de Montréal.

PICARD, M. (1982a) : " $\hat{A}$ la recherche de l'épenthèse en québécois", dans Recherches linguistiques à Montréal, vol. 18 .

PICARD, M. (1982b) : "The Latin Negative Prefixe in-", dans Recherches linguistiques à Montréal, vol. 18.

PIQUETTE, E. (1976) : l'Adverbe de temps en français : en quoi la syntaxe appuie la sémantique, mémoire de maîtrise, Université du Québec à Montréal.

Petit Robert, Dictionnaire de la langue française (1979).

ROBERT ET COLLINS (1981) : Dictionnaire français-anglais, English-French Dictionary.

SABOURIN, C. (1975), l'Adverbe de phrase : essai de caractérisation par une méthode statistique, mémoire de maîtrise, Université du Québec à Montréal.

SABOURIN, C. et J. CHANDOUX (1977) : l'Adverbe français : essai de catégorisation, Documents de linguistique quantitative, Université Paris VI, Association Jean Favard.

TARDIF, M. (1982) : "La sous-catégorisation des adverbes en -ment", dans Recherches linguistiques à Montréal, vol. 18. 